\title{
Determination of anaerobic threshold in rats using the lactate minimum test
}

F.A. Voltarelli,
C.A. Gobatto and
M.A.R. de Mello

\section{Correspondence}

M.A.R. de Mello

Departamento de Educação Física

IB, UNESP

Avenida 24-A, 1515

13506-900 Rio Claro, SP

Brasil

Fax: +55-19-534-0009

E-mail: mellomar@rc.unesp.br

Presented at the XVII Annual Meeting of the Federação de Sociedades de Biologia Experimental, Salvador, BA, Brazil, August 28-31, 2002.

Research supported by FAPESP (Nos. 96/05425-2, 01/03356-3 and 95/5778) and CNPq (No. 522755/96-8).

Received April 8, 2002 Accepted August 27, 2002

Abstract

The break point of the curve of blood lactate $v$ s exercise load has been called anaerobic threshold (AT) and is considered to be an important indicator of endurance exercise capacity in human subjects. There are few studies of AT determination in animals. We describe a protocol for AT determination by the "lactate minimum test" in rats during swimming exercise. The test is based on the premise that during an incremental exercise test, and after a bout of maximal exercise, blood lactate decreases to a minimum and then increases again. This minimum value indicates the intensity of the AT. Adult male (90 days) Wistar rats adapted to swimming for 2 weeks were used. The initial state of lactic acidosis was obtained by making the animals jump into the water and swim while carrying a load equivalent to $50 \%$ of body weight for 6 min (30-s exercise interrupted by a 30-s rest). After a 9min rest, blood was collected and the incremental swimming test was started. The test consisted of swimming while supporting loads of 4.5, 5.0, 5.5, 6.0 and $7.0 \%$ of body weight. Each exercise load lasted $5 \mathrm{~min}$ and was followed by a 30 -s rest during which blood samples were taken. The blood lactate minimum was determined from a zerogradient tangent to a spline function fitting the blood lactate $v s$ workload curve. AT was estimated to be $4.95 \pm 0.10 \%$ of body weight while interpolated blood lactate was $7.17 \pm 0.16 \mathrm{mmol} / \mathrm{l}$. These results suggest the application of AT determination in animal studies concerning metabolism during exercise.

In human subjects blood lactate concentration increases exponentially with exercise intensity. The break point on the curve of blood lactate vs workload during incremental exercise has been termed blood lactate threshold or anaerobic threshold (AT) (1), although anaerobiosis as a cause of the accelerated lactate production has been questioned (2). AT occurs within the range of submaximal exercise intensities, in general between 50 and $80 \%$ of the maximal load and at a blood lactate concentration of ap- proximately $4.0 \mathrm{mmol} / \mathrm{l}$ (1)

Despite many attempts $(1,2)$, a physiological basis for AT has not yet been fully established. Nevertheless, the threshold determinations are useful for the assessment of subject endurance capacity. Today, AT measurements are very popular for the evaluation of training effectiveness in athletes, assuming that the higher the threshold, the greater the endurance capacity $(3,4)$. Determinations of AT have also proved to be of value in clinical studies (5). 
In 1993, Tegtbur et al. (6) reported the development of a test named lactate minimum test to determine AT. The test involved subjects performing a short period of supramaximal exercise to induce hyperlactemia before starting a standard incremental treadmill test. The blood lactate minimum (BLM) was defined as the velocity at which a curve fitted to the U-shaped blood lactate data derived from the incremental test reached a nadir. This minimum lactate point was supposed to indicate the lactate threshold (7). After the initial proposal, this test was investigated extensively in human subjects, since it seemed to offer advantages over existing methods for AT estimation. Unlike the subjective selection of threshold points as done for lactate threshold and ventilatory threshold determinations, a procedure that has been criticized for poor inter-reviewer reliability (8), BLM can be determined objectively by applying a zero-gradient tangent to a spline function fitting the blood lactate response to the incremental exercise test (7). Also, in contrast to methods utilizing reference blood lactate concentrations, such as the onset of blood lactate accumulation at $4.0 \mathrm{mmol} / \mathrm{l}$ blood lactate, the BLM is apparently unaffected by conditions of glycogen depletion (6). The lactate minimum test has been considered to be sensitive to changes in endurance capacity after aerobic exercise training in some circumstances (9-11), but not in others $(7,12,13)$.

For obvious reasons, a significant number of studies involving exercise have been conducted on laboratory animals, mainly rats, and blood lactate concentration was used in many for the determination of effort intensity. However, this procedure is limited by the small amount of information available concerning lactate kinetics during exercise in rats. To our knowledge, only few studies dealing with lactate threshold determinations in rats have been reported in the literature, most of them using treadmill running as an ergometer $(14,15)$. Only one study analyzed lactate kinetics during swimming (16). Thus, the present study was designed to analyze blood lactate alterations during an incremental swimming exercise test performed after a bout of supramaximal exercise in rats to determine whether the AT can be detected in the animals under these conditions.

All experiments involving the animals were conducted according to the policy of the American College of Sport Medicine on Research with Experimental Animals. Adult (90-120 days) male Wistar rats $(\mathrm{N}=10)$ were used. The animals were fed a commercial chow for rodents (Labina, Purina, Campinas, SP, Brazil) ad libitum and had free access to water throughout the experimental period. The animals were housed in collective cages ( 5 rats per cage) at $25^{\circ} \mathrm{C}$ in a room with lights on from 6:00 to 18:00 h. All rats were adapted to water before the beginning of the tests. The adaptation consisted of keeping the animal in shallow water at $31 \pm$ $1^{\circ} \mathrm{C}$ (17) for $60 \mathrm{~min}, 5$ days/week for 2 weeks. The purpose of the adaptation was to reduce stress without promoting exercise training adaptations.

The incremental exercise test protocol employed was based on the lactate minimum test described by Tegtbur et al. (6) for human subjects, adapted to rats. The lactate minimum test consists of performing a short period of supramaximal exercise to induce hyperlactemia before starting an incremental exercise test. In order to produce hyperlactemia, the rats were induced to jump into the water for $6 \mathrm{~min}$ (30-s exercise interrupted by 30 -s rest) carrying a load (lead fish sinkers strapped to the chest) of 50\% body weight in a cylinder tank $50 \mathrm{~cm}$ deep and $25 \mathrm{~cm}$ in diameter, filled with water at $31 \pm 1^{\circ} \mathrm{C}$. After a 9-min recovery interval, the rats were submitted to an incremental test consisting of swimming while carrying loads of $4.5,5.0$, 5.5, 6.0 and $7.0 \%$ of body weight. Exercise at each load lasted $5 \mathrm{~min}$ and was followed by a 30 -s rest during which blood samples were taken for lactate measurement. The 
BLM (the load corresponding to the nadir of the blood lactate $v s$ workload, as a \% of body weight plot) was estimated from the zerogradient tangent to a spline function fitting the exercise blood lactate data. This minimum value corresponded to the AT. The adequate interval ( $9 \mathrm{~min}$ ) between the end of the supramaximal exercise and the beginning of the incremental exercise test was established in a separate set of experiments performed with the same rats one week before. For this purpose, the rats were induced to jump into the water for $6 \mathrm{~min}$ as described above and blood samples were collected for lactate determination $6,7,8,9,10$ and 11 min after the end of the exercise bout. Peak blood lactate occurred at the 9th min (data not shown). Thus, we adopted a 9-min recovery time before the beginning of the incremental test.

Since it is often assumed that the aerobic/ anaerobic transition is demarcated by the higher exercise intensity at which blood lactate does not increase beyond the initial transient during a constant load exercise, i.e., a maximal lactate steady state (MLSS) level (1), we also checked for blood lactate stabilization at the load corresponding to the threshold obtained by the lactate minimum test. One week after the lactate minimum test, the rats were submitted to swimming exercise while carrying constant loads below BLM ( $4.0 \%$ of body weight $)$ corresponding to BLM (5.0\% of body weight, see Figure 1) and above BLM (6.5\% of body weight) in $100 \times 80 \times 80 \mathrm{~cm}$ tanks, subdivided into four cylinder compartments filled with water at $31 \pm 1^{\circ} \mathrm{C}$. This device permitted the simultaneous evaluation of four animals swimming individually. Each animal participated in three experimental tests with a 3day interval between them. The sequence of loads was distributed at random and the same load was never used twice by the same animal. Each test consisted of continuous swimming for 30 min with one load. After each 5-min period of exercise, blood samples were collected for lactate determination. The loads of 4.0 and $6.5 \%$ were chosen because in a previous study we demonstrated that sedentary rats showed MLSS during swimming exercise with overloads of 5.0 and $6.0 \%$ of body weight (16). The data obtained in this set of experiments were analyzed by one-way ANOVA. When necessary, the Newman-Keuls post hoc comparison test was used. In all cases, statistical significance was set at $\mathrm{P}<0.05$.

All blood samples $(25 \mu \mathrm{l})$ were collected from a cut at the tip of the tail with the aid of graded capillary tubes during the exercise tests and placed in 1.5-ml Eppendorf tubes containing $50 \mu \mathrm{l} 1 \%$ sodium fluoride. To avoid blood lactate dilution with residual water in the tail of the animal, the rats were dried with a towel immediately before blood collection. The lactate concentrations were determined with a lactate analyzer (YSI Model 1500 SPORT, Yellow Springs, OH, USA).

Blood lactate concentrations for a single rat at the consecutive workloads during the lactate minimum test are presented in Figure 1. The curve fitted to the blood lactate data derived from the incremental load swimming test presented, as expected, a U-shaped form. The BLM, $5.2 \%$, is indicated by the load at which this curve reached its nadir. In this particular case, the estimated BLM was $5.2 \%$ of body weight load, while the interpolated blood lactate concentration was 7.0 $\mathrm{mmol} / \mathrm{l}$. Analysis of individual curves permitted us to determine the AT. The mean AT, expressed as the load at which BLM was

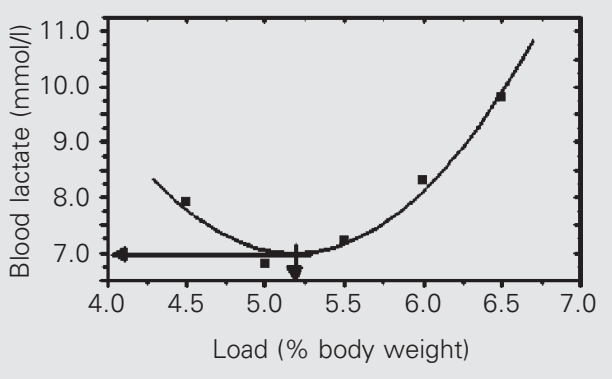

Figure 1. Determination of the blood lactate minimum (BLM) of a single rat during an incremental swimming exercise test. Each point indicates blood lactate concentration $(\mathrm{mmol} / \mathrm{l})$ on the ordinate after a 5-min exercise bout with the animal supporting each load indicated on the abscissa. The BLM and the respective workload were calculated using the spline function. The vertical arrow indicates the estimated workload corresponding to BLM while the horizontal arrow indicates the interpolated blood lactate concentration. 
estimated in all rats, was $4.95 \pm 0.10 \%$ of body weight, while the mean interpolated blood lactate concentration at this load was $7.17 \pm 0.16 \mathrm{mmol} / \mathrm{l}$.

Figure 2 presents mean blood lactate concentrations during the constant load exercise tests. The animals showed blood lactate stabilization with the load below BLM (4.0\% of body weight, Figure $2 \mathrm{~A}$ ), at $5.06 \pm 0.25$ $\mathrm{mmol} / \mathrm{l}$ blood lactate and with the load corresponding to the BLM (5.0\% of body weight, Figure 2B), at $6.41 \pm 0.30 \mathrm{mmol} / 1 \mathrm{blood}$ lactate. There was a progressive increase in blood lactate concentrations with load above the BLM (6.5\% of body weight, Figure 2C). The mean estimated blood lactate concentration at BLM was significantly higher $(\mathrm{P}<0.05, t$-test $)$ than the mean blood lactate observed during the constant load test at $5.0 \%$ of body weight.

The objective of this study was to establish a protocol of swimming exercise with progressive intensities for rats based on the lactate minimum test proposed for human subjects $(6,7)$ in order to determine their AT. This test involves a) a short period of supramaximal exercise to induce hyperlactemia, b) a brief recovery period to assure high levels of lactemia, and c) a test protocol of incremental exercise.

Despite the limited information on the theoretical basis for the lactate minimum concept, this concept was found to be useful for AT determination in human subjects in some circumstances (9), and appeared attractive for application to laboratory rats. The only procedure previously demonstrated to be adequate to estimate $\mathrm{AT}$ in swimming rats requires several tests performed during more than two weeks (16). This impairs its application under some special conditions such as pregnancy and lactation, which last only three weeks in the rat, and in tumorbearing animals. Since the lactate minimum test requires only one day, it would represent an alternative procedure for AT determination in swimming rats, if it proved to be well tolerated by the animals.

In the test originally described for human subjects, the initial lactic acidosis was induced in athletes by two successive exhausting runs. The second run was followed by an 8-min recovery (slow walking). Soon after, the incremental test began (6). In a previous study we demonstrated that sedentary male Wistar rats, immediately after five sessions of jumps into the water while carrying a load of $50 \%$ of body weight, $30 \mathrm{~s}$ of exercise interrupted by $1 \mathrm{~min}$ of rest, show blood lactate concentrations of $5.0 \pm 0.7 \mathrm{mmol} / 1$. After 10 sessions of the same exercise, the values rose to $7.2 \pm 1.1 \mathrm{mmol} / \mathrm{l}(18)$. On the basis of this information, to obtain the initial acidosis, in the present study the rats were induced to jump into water while carrying an overload of $50 \%$ of body weight. The rats did not show difficulty in performing the protocol and hyperlactemia appeared, confirming our previous findings (18). The loads used to establish the incremental swimming test protocol were based on another study by our group (16) in which we examined blood lactate kinetics during constant load swimming exercise in male Wistar rats. $4.0 \%$ of body weight); $B$, equal to BLM $(5.0 \%$ of body weight), and $C$, above BLM $(6.5 \%$ of body weight). Observe the blood lactate stabilization with the $4.0 \%$ load $(5.06 \pm 0.25 \mathrm{mmol} / \mathrm{l})$ and the $5.0 \%$ load $(6.41 \pm 0.30$ $\mathrm{mmol} / \mathrm{ll}$. Data are reported as means \pm SD for 10 rats per group. In each panel, each different letter indicates significant differences in blood lactate from the other values $(P<0.05$ ANOVA).
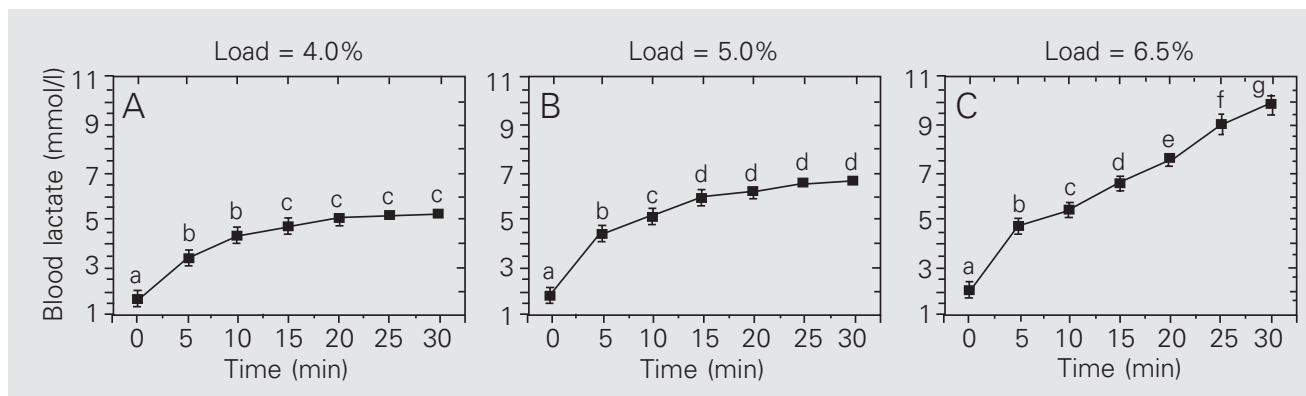
The analysis of our lactate minimum test results demonstrated that the blood lactate alterations occurring in rats during incremental exercise show a pattern similar to that described for human subjects. This permitted us to determine the AT, expressed as the load at which the BLM point was observed in all rats $(4.95 \pm 0.10 \%$ of body weight), while the mean interpolated blood lactate concentration at this load was $7.17 \pm$ $0.16 \mathrm{mmol} / \mathrm{l}$. In a previous study on rats, Pillis et al. (14) observed that during an incremental treadmill run test, AT occurred when the blood lactate concentration was approximately $4.0 \mathrm{mmol} / \mathrm{l}$. This suggests that the type of exercise used (running or swimming) may affect the value of blood lactate at AT.

According to Mader and Heck (1), the AT is a term that refers to the oxygen consumption during exercise above which the rate of lactate production exceeds the rate at which it can be removed, thus causing an increase in blood lactate. The highest exercise intensity at which blood lactate does not increase, therefore, should be equivalent to the AT. Thus, a second question that was addressed in the present investigation was the following: was there blood lactate stabilization during constant load exercise at the load corresponding to the BLM estimated by the lactate minimum test? Our data show that rats were able to keep a stable blood lactate at workloads below $(4.0 \%$ of body weight) and equivalent to $(5.0 \%$ of body weight) the estimated BLM. At the load above the estimated BLM $(6.5 \%$ of body weight), blood lactate increased with time.
This indicates a stable entry/removal ratio at the BLM workload estimated by the lactate minimum test and a higher production in relation to removal at the load above the BLM. Since in a previous study we demonstrated that the lactate entry/removal in sedentary adult male Wistar rats submitted to acute swimming reached a maximum at workloads between 5.0 and $6.0 \%$ of body weight (16), it is quite possible that the BLM estimated by the lactate minimum test employed here corresponds to the MLSS.

We also checked if there was a coincidence between blood lactate concentration at the BLM estimated by the lactate minimum test and the blood lactate concentrations observed during constant load exercise while carrying the BLM load. The mean blood lactate concentrations observed during constant load exercise supporting a load of $5.0 \%$ body weight were considered to be significantly lower than the value of blood lactate at the BLM estimated by the lactate minimum test. This supports the hypothesis that the value of blood lactate concentration at the AT may be protocol dependent. A similar observation was reported by Jones and Doust (7) working with human subjects.

Using the lactate minimum test protocol described in the present study, it was possible to determine AT in rats during swimming exercise. These results suggest a potential application of AT in animal studies concerning metabolism during exercise. However, before this application, more studies are required in order to establish whether or not the blood lactate concentration at AT is protocol dependent in rats.

\section{References}

1. Mader A \& Heck $H$ (1986). A theory of metabolic origin of the anaerobic threshold. International Journal of Sports Medicine, 7: 45-65.

2. Brooks GA (2000). Intra- and extracellular lactate shuttles. Medicine and Science in
Sports and Exercise, 32: 790-799

3. Jones AM \& Carter $H$ (2000). The effect of endurance training on parameters of aerobic fitness. Sports Medicine, 29: 373376.

4. Billat LV (2000). Interval training for per- formance: scientific and empirical practice-special recommendations for middle and long-distance running, part I: aerobic interval training. Sports Medicine, 31: 1331.

5. Wasserman K \& Mcllroy MB (1964). De- 
tecting the threshold of anaerobic metabolism in cardiac patients during exercise. American Journal of Cardiology, 14: 844-852.

6. Tegtbur U, Busse MW \& Braumann KR (1993). Estimation of individual equilibrium between lactate production and catabolism during exercise. Medicine and Science in Sports and Exercise, 25: 620627.

7. Jones AM \& Doust JH (1998). The validity of the lactate minimum test for determination of the maximal lactate steady state and physiological correlates to $8 \mathrm{~km}$ running performance. Medicine and Science in Sports and Exercise, 30: 1304-1313.

8. Gladden LB, Yates JW, Stremel RW \& Stamford BA (1985). Gas exchange and lactate anaerobic thresholds: inter- and intra-evaluator agreement. Journal of Applied Physiology, 58: 2082-2089.

9. Tegtbur U, Machold H, Meyer H, Storp D \& Busse MW (2001). Determining the extent of extensive physical performance in patients with coronary heart disease. Zeitschrift für Kardiologie, 90: 637-645.

10. Bacon L \& Kern M (1999). Evaluating a test protocol for predicting maximum lactate steady state. Journal of Sports Medicine and Physical Fitness, 39: 300-308.

11. Simões HG, Campbell CSG, Tango $\mathrm{MH}$, Mello F, Maziero DC \& Baldissera V (2000). Lactate minimum test in swimming: relationship to performance and maximal lactate steady state. Medicine and Science in Sports and Exercise, 32: S161 (Abstract).

12. Carter H, Jones AM \& Doust JH (1999). Effect of 6 weeks of endurance training on lactate minimum speed. Journal of Sports Sciences, 17: 957-967.

13. Carter H, Jones AM \& Doust JH (1999). Effect of incremental protocol on the lactate minimum speed. Medicine and Science in Sports and Exercise, 31: 837-845.

14. Pillis W, Zarzeczny R, Langfort J, KaciubaUscilko H, Nazar K \& Wojtyna J (1993). Anaerobic threshold in rats. Comparative
Biochemistry and Physiology, 106A: 285289.

15. Langfort J, Zarzeczny R, Pillis W, KaciubaUscilko H, Nazar K \& Porta S (1996). Effects of sustained hyperadrenalinemia on exercise performance and lactate threshold in rats. Comparative Biochemistry and Physiology, 114A: 51-55.

16. Gobatto CA, Mello MAR, Sibuya CY, Azevedo JRM, Santos LA \& Kokubun E (2001). Maximal lactate steady state in rats submitted to swimming exercise. Comparative Biochemistry and Physiology, 130: 21-27.

17. Harri M \& Kuusela $P$ (1986). Is swimming exercise or cold exposure for rats? Acta Physiologica Scandinavica, 126: 189-197.

18. Silva MP, Marcondes MCG \& Mello MAR (1999). Exercício aeróbio e anaeróbio: efeitos sobre a gordura sérica e tecidual de ratos alimentados com dieta hiperlipídica. Revista Brasileira de Atividade Física e Saúde, 4: 43-56. 